\title{
Abuso Físico Infantil: Analisando o Estresse Parental e o Apoio Social ${ }^{1}$
}

\author{
Lílian Paula D. Bérgamo² \\ Marina Rezende Bazon \\ Universidade de São Paulo
}

\begin{abstract}
RESUMO - Verificou-se se o estresse parental e o apoio social relacionar-se-iam ao abuso físico infantil, comparando-se G1 cuidadores notificados por abusos, e G2 - não-agressores. Utilizou-se um Questionário de caracterização socio-demográfica e econômica e um sobre Apoio Social e o Índice de Estresse Parental. Obteve-se diferença significativa no escore geral de estresse e na dimensão Criança Difícil, com G1 vivenciando mais estresse. Ademais, G1 apresentou menor nível de apoio, no geral e nas dimensões Afetiva e de Interação Social Positiva. G1 também relatou menos satisfação com o bairro de residência e, em média, era mais jovem quando do nascimento do primeiro filho. Essas variáveis devem receber investimentos em programas de prevenção e de tratamento dirigidos a tal problemática.
\end{abstract}

Palavras-chave: abuso físico; estresse parental; apoio social e maus-tratos.

\section{Physical Child Abuse: Analyzing Parental Stress and Social Support}

\begin{abstract}
This study verified the existence of a relationship between parental stress, social support, and physical child abuse. Two groups were compared, G1 - caregivers reported for abuse, and G2 - caregivers not reported for abuse. Three questionnaires were applied: one assessing social demographic and economic variables, one measuring Social Support, and one assessing the Parental Stress Index. Results indicated that G1 not only showed higher levels of stress and lower levels of social support, but also lower values on the dimensions "Affective" and "Positive Social Interaction". The parents of G1 were younger when their first child was born, and reported less satisfaction with their neighborhood. The investigated variables should be incorporated in prevention and treatment programs directed to reduce physical child abuse.
\end{abstract}

Keywords - physical abuse; parental stress; social support; maltreatment.

A violência contra crianças e adolescentes praticada por pais ou cuidadores é um fenômeno altamente prevalente em vários países, incluindo o Brasil. Nesse contexto, destacam-se os abusos físicos por conta de sua visibilidade, considerados a forma mais evidente de maus-tratos infantis, devido à probabilidade de resultar em marcas ou lesões corporais que, por vezes, constituem uma emergência médico-social, com elevado impacto psicológico (Sacroisky, 2003).

A OMS e a Sociedade Internacional para a Prevenção do Abuso e da Negligência Infantil (OMS \& ISPCAN, 2006), em um esforço de síntese das principais proposições veiculadas, com vistas à superação de diferenças culturais envolvendo a conceituação do problema, sugerem que os abusos físicos sejam concebidos como "o uso intencional da força física contra a criança que resulta, ou tem alta possibilidade de resultar, em dano a sua saúde, sobrevivência, desenvolvimento e dignidade" (p.10). Observa-se que essa definição amplia o rol de situações passíveis de serem classificadas como abusivas no sentido de abarcar não somente as ações que deixam marcas e/ou ferimentos no corpo, mas as que, sem fazer isso, afetam o desenvolvimento psicológico/emocional da criança.

1 Apoio CAPES. Este trabalho faz parte da Dissertação de Mestrado desenvolvida no Departamento de Psicologia e Educação da FFCLRPUSP, intitulada "Maus-tratos físicos de crianças: contribuições para a avaliação de fatores de risco psicossociais", de autoria da primeira autora com orientação da segunda.

2 Endereço para correspondência: Departamento de Psicologia e Educação. Faculdade de Filosofia Ciências e Letras de Ribeirão Preto. Universidade de São Paulo. Av. Bandeirantes, 3900. Ribeirão Preto, SP. CEP: 14040-901. Fone: (16) 3602-3830. Fax: (16) 3602-4835. E-mail: mbazon@ffclrp.usp.br, li.bergamo@pg.ffclrp.usp.br
É importante sublinhar que os atos abusivos contra as crianças podem ocorrer em meio às práticas educativas (de punição corporal) implementadas pelos cuidadores como forma de corrigir determinados comportamentos infantis, sendo que alguns autores defendem a concepção de uma linha tênue entre um fenômeno e outro (Carmo \& Harada, 2006; Weber, Viezzer \& Brandenburg, 2004;). No entanto, apesar de as práticas educativas mais duras, baseadas na punição corporal, se constituírem em um importante fator para o abuso físico de crianças, a etiologia do fenômeno implica na identificação e na compreensão da dinâmica de outros fatores para que se consiga fazer o seu diagnóstico e planejar ações adequadas de prevenção e de tratamento.

Nesse sentido, diversas pesquisas têm empreendido esforços para identificar fatores de risco específicos, associados aos abusos físicos, sendo esses fatores ligados às características do adulto cuidador, da criança e do contexto de vida destes (Ateah \& Durrant, 2005; Balge \& Milner, 2000; Bordin, Paula, Nascimento \& Duarte, 2006; Cecconello, De Antoni \& Koller, 2003; De Antoni, Barone \& Koller, 2007; De Paúl \& Domenech, 2000; Montes, De Paul \& Milner, 2001; Palusci, Smith \& Paneth, 2005; Reichenheim, Dias \& Moraes, 2006; Woodward $\&$ Fergusson, 2002). O presente trabalho vem contribuir neste panorama, investigando particularmente o papel de dois fatores apontados pela literatura como sendo de risco para os abusos físicos: o estresse parental e o apoio social, sendo esses descritos como contextuais nas pesquisas que tratam do tema (Crouch \& Behl, 2001; Rodriguez \& Richardson, 2007).

Com relação ao estresse parental, tem-se observado que cuidadores que o experienciam em maior nível tem a 
possibilidade de utilizar mais comportamentos de controle e de punição nas interações com os filhos, caracterizando um estilo de disciplina autoritário, o que parece incrementar a probabilidade de haver abusos físicos (Rodriguez \& Richardson, 2007; Rodriguez, 2010).

Muitos dos estudos trabalham com a concepção de que a totalidade de estresse vivida pelos cuidadores na interação com a criança decorre de certas características de ambos, assim como de variáveis contextuais ligadas diretamente ao exercício do papel parental (Combs-Orme, Cain \& Wilson, 2004; Dessen \& Szelbracikowski, 2004, 2006; Jackson, 2000; Rodriguez $\&$ Richardson, 2007). Outros estudos, contudo, apontam que a fonte principal do estresse vivido nesse tipo de interação provém da percepção que o cuidador tem das características da criança, bem como do seu comportamento (DiLauro, 2004; Dopke \& Milner, 2000). Dopke e Milner (2000), por exemplo, avaliando o estresse parental vivido nas interações com as crianças, especificamente quando essas manifestam comportamentos considerados inadequados ou de desobediência, apontaram que as mães classificadas como de alto risco para o abuso, em comparação com as de baixo risco, percebiam a desobediência como uma situação mais estressante por ser, em suas concepções, altamente ameaçadora e incontrolável.

O estresse parental tem sido apontado como uma variável importante na dinâmica das famílias com crianças que efetivamente apresentam algum tipo de problema de comportamento (Jackson, 2000). No Brasil, estudos também identificaram uma relação entre o estresse parental e problemas de comportamento exteriorizado em crianças, sendo que a manifestação destes, por sua vez, estaria relacionada a práticas de punição física e verbal, entre outros fatores (Dessen \& Szelbracikowski, 2004, 2006). Diante dessas considerações é possível imaginar um ciclo no qual o estresse parental se constitui em um dos fatores causais de práticas coercivas de disciplina que, por sua vez, seria um dos fatores geradores de problemas de comportamento infantis, os quais incrementariam o nível de estresse experimentado e o risco de abusos físicos.

Ademais, com base nos resultados das investigações de Dopke e Milner (2000) e DiLauro (2004), onde altos níveis de estresse estariam atrelados, principalmente, à percepção negativa de comportamentos/características da criança, pode-se pensar na existência de uma interação entre aspectos do funcionamento cognitivo dos cuidadores com os níveis de estresse experimentados para a composição do risco de abuso. A interação da variável estresse com variáveis pertencentes ao domínio cognitivo do cuidador tem sido investigada por meio da aplicação do Modelo do Processamento da Informação Social, em casos de abuso físico, o que tem indicado que déficits nos processos cognitivos seriam influenciados por fatores contextuais como, por exemplo, o estresse (Milner, 2003). Nesse sentido, defende-se que as variáveis contextuais contam mais para compor o risco de abuso que os próprios processos cognitivos em si (Crouch \& Behl, 2001; Rodriguez \& Richardson, 2007; Rodriguez, 2010). Crouch e Behl (2001), por exemplo, verificaram que o nível de estresse foi positivamente relacionado com o risco de abuso físico independente de haver ou não crenças relacionadas à eficácia da punição corporal como prática educativa, as quais fariam parte dos esquemas cognitivos dos cuidadores.
Paralelamente, a literatura aponta que em etapas de transição e mudanças do sistema familiar, nas quais normalmente se experimenta maior estresse, como por exemplo, por ocasião do nascimento de filhos, dispor de uma rede de apoio social e poder contar com ajuda (principalmente a oriunda das pessoas próximas, como a do parceiro e a da família estendida), é considerado um dos fatores mais relevantes na moderação do estresse, pois contribui para a manutenção do equilíbrio das relações familiares, bem como para o seu funcionamento adaptativo (Dessen \& Braz, 2000).

Tratando especificamente da relação cuidador-criança, altos níveis percebidos de auto-eficácia parental e de apoio social foram identificados como fatores protetores contra o estresse, já que gerariam nos cuidadores um sentimento de maior controle das situações, principalmente nas quais houvesse a manifestação de problemas de comportamento pela criança, porque a avaliação positiva da ajuda recebida proporcionaria condições para a resolução de problemas, especificamente no que respeita às relações com os filhos (Cecconello \& cols., 2003; De Antoni \& cols., 2007; Jackson, 2000; Suzuki, 2010). Mesmo determinados fatores de risco de natureza ontogenética, como a maternidade precoce (antes dos 20 anos de idade) (Budd, Heilman \& Kane, 2000; Woodward \& Fergusson, 2002), e o histórico de maus-tratos do próprio cuidador, na sua infância, frente à oferta de apoio social e à percepção de sua adequação às necessidades experimentadas, teriam seu impacto absolutamente diminuído (Crouch, Milner \& Thomsen, 2001; McLewin \& Muller; 2006; Lee, 2009; Zelenko, Huffman, Lock, Kennedy \& Steiner, 2001).

Por outro lado, estudos verificaram que o isolamento social ou a falta de uma rede de apoio social e afetivo faria aumentar ainda mais o risco de práticas abusivas com os filhos (Bigras \& Paquete, 2000; Cecconello \& cols., 2003; De Antoni \& cols., 2007; Hecht \& Hansen, 2001; Ochotorena \& Madariaga, 1996; Suzuki, 2010). Bigras e Paquete (2000), por exemplo, notaram que a qualidade da interação mãe-criança apresenta-se inversamente relacionada ao isolamento social percebido, sendo que a ansiedade do cuidador parece tanto mais elevada quanto mais forte é a percepção de não poder contar com ajuda, em caso de necessidade.

Cumpre considerar, segundo Coohey (1996), que o apoio social, além de englobar o conceito de redes formais e informais de ajuda, e o número de contatos efetivamente realizados com as pessoas que as compõem, engloba também os elementos de percepção quanto ao apoio proveniente de cada membro da rede ("apoio percebido") e a natureza do apoio oferecido (se baseado em ajuda emocional e/ou instrumental e/ou material - "tipo de apoio recebido"). Segundo a autora, mães que cometem abuso físico geralmente reportam um baixo nível de apoio emocional (sentindo não terem quem as ouça, quem as ajude na tomada de decisões e quem as compreenda) e também instrumental (sentindo não poderem contar com pessoas que as ajudem no cuidado com a criança). O número de pessoas a compor as redes de apoio e de contatos realizados com estas, bem como o apoio do tipo material/financeiro não se mostraram um diferencial nessas mães.

No tocante às redes formais de ajuda, constituída por pessoas/profissionais de organismos comunitários e/ou instituições, a literatura tem apontado a fragilidade da integração 
social de cuidadores envolvidos em situações de maus-tratos nesse tipo de rede devido, principalmente, à presença de atitudes e sentimentos negativos desses com relação ao entorno comunitário, o que foi verificado, inclusive, em diferentes culturas (Gracia \& Musito, 2003). Guterman, Lee, Taylor e Rathouz (2009) acrescentam que a percepção negativa do entorno comunitário, como por exemplo, a da inexistência de suporte e de coesão social, relacionar-se-ia indiretamente com o risco dos adultos para o cometimento de maus-tratos, já que essa relação seria mediada pela sensação de exercer pouco controle sobre os eventos no entorno, o que produz um alto nível de estresse parental, que por sua vez, seria um preditor proximal do risco de maus-tratos.

Considerando o panorama descrito, com base nos apontamentos da literatura, quanto ao papel do estresse parental e o da falta de apoio social (isolamento), como importantes fatores de risco para o abuso físico de crianças, no presente estudo buscou-se verificar se efetivamente estas duas variáveis encontravam-se associadas ao fenômeno, em nosso contexto. Hipotetizou-se que cuidadores com histórico de abuso físico contra os filhos apresentariam níveis mais elevados de estresse parental e disporiam, bem como perceberiam, menor nível de apoio social, se comparados a um grupo controle.

\section{Método}

Para o desenvolvimento da investigação, optou-se por um delineamento de estudo de casos clínicos e controles, com vistas à comparação dos grupos nas variáveis privilegiadas. Esse foi avaliado por Comitê de Ética em Pesquisa com Seres Humanos.

\section{Participantes}

Participaram do estudo 60 cuidadores, 30 constituíram o grupo clínico (G1), por terem sido notificados ao Conselho Tutelar por abuso físico contra os filhos, e 30 constituíram o grupo de comparação (G2), por não apresentarem relato de maus-tratos. Os participantes do G1 foram selecionados com base nos registros oficiais dos Conselhos Tutelares da cidade de Ribeirão Preto/SP, retendo-se aqueles casos que correspondiam a abuso físico, segundo a definição adotada no presente estudo, relativos a crianças/adolescentes com até 15 anos de idade, visto que esse tipo de abuso é mais raro em idades superiores a essa. Privilegiou-se as notificações feitas a no máximo três anos antes do ano da coleta de dados, com vistas a aumentar as chances de encontrar as famílias nos mesmos endereços registrados e de a situação sócio-familiar atrelada ao abuso não ter sofrido grandes alterações.

Do total dos casos selecionados (90), houve perdas significativas por razão de mudanças de endereço e/ou endereços não localizados (44 casos) e algumas recusas (8). Outros oito casos não foram contatados em função de a amostra ter atingido o número pretendido para a composição de $\mathrm{G} 1(\mathrm{n}=30)$.

Os participantes que compuseram o G2 foram recrutados a partir da indicação de profissionais (psicólogos e assistentes sociais) atuando na área da assistência social, nas comunidades da mesma cidade, seguindo o critério de os mesmos não possuírem histórico de abuso físico contra os filhos. Ainda, para a constituição de G2, procurou-se pelo pareamento, em relação a G1, nos seguintes aspectos: idade, sexo, escolaridade, nível sócio-econômico, situação conjugal, total de crianças sob seus cuidados e idade de um dos filhos (a criança que foi notificada ao Conselho Tutelar). Para tanto, 40 pessoas foram contatadas para se conseguir o total de 30 participantes, sendo que quatro recusaram colaborar com o estudo e seis, embora tendo colaborado, após uma análise preliminar dos dados coletados, tiveram seus dados descartados para o presente estudo por não atenderem aos critérios de pareamento.

Os participantes do presente estudo caracterizam-se por terem em média 34 anos em G1 e 36 anos em G2 ( $p=0,373)$; $20 \%(n=6)$ serem do sexo masculino e $80 \%(n=24)$ do feminino, em ambos os grupos; $70 \%(\mathrm{n}=21)$ possuírem companheiro(a) também em ambos os grupos; terem escolaridade média de 6,2 anos em G1 e 6,5 anos em G2 (p=0,765). Os participantes de G1 tinham em média 2,9 filhos com média de idade de 9,7 anos, e em G2 o número médio de filhos era de 2,5 com média de idade de 9,2 anos ( $\mathrm{p}=0,363$ e $\mathrm{p}=0,603$, respectivamente). Em ambos os grupos, os participantes, eram majoritariamente de grupos familiares pertencentes à classe $\mathrm{C}(\mathrm{G} 1=57 \%$ e $\mathrm{G} 2=63 \%, \mathrm{p}=0,117)$, que é a classe mais numerosa no Brasil, na qual a renda familiar estimada gira em torno de 927 reais. Nessa direção, verificou-se também que a razão do número de pessoas constituindo a família e co-habitando por número de cômodos da residência não apresentou diferença significativa nos grupos, sendo essa de 1,05 para G1e de 0,97 para G2.

Frisa-se, entretanto, que mesmo tendo-se conseguido trabalhar com participantes muito semelhantes em termos sócio-demográficos, uma diferença significativa marcou os dois grupos: a "idade do responsável por ocasião do nascimento do primeiro filho" ( $t=729,50, p \leq 0,01)$. Os participantes do G1 eram em média mais jovens na ocasião em que tiveram o primeiro filho $(19,90 \pm 5,65)$ que os participantes do G2 $(23,23 \pm 5,04)$, observando-se o fato de terem tido seu primeiro filho, em média, antes dos vinte anos de idade, ao passo que em G2 essa experiência teria acontecido depois dos vinte anos.

\section{Instrumentos}

Questionário de Caracterização Sócio-demográfica da família: elaborado com base no modelo proposto por Bringiotti (1999), visa a obtenção de informações possibilitando uma caracterização do respondente em termos de idade, sexo, nível educacional, estado civil, além de buscar por informações sobre a idade no momento do nascimento do primeiro filho, número de filhos ou crianças sob os cuidados, idade das crianças, bem como sobre determinadas condições de vida, como número de moradores por número de cômodos da casa e impressão do bairro de residência/satisfação com a comunidade, por serem esses elementos apontados na literatura especializada como indicadores de risco para os maus-tratos infantis.

Critério de Classificação Econômica Brasil - CCEB: desenvolvido a partir de um estudo realizado com base no banco de dados do -"Levantamento Sócio-Econômico" 
(LSE), de 1993, do IBOPE (ABEP, 2003), caracteriza-se como um check-list por meio do qual verifica-se a posse de determinados objetos ou bens de consumo e o grau de instrução do chefe da família, de modo a poder classificar a família, em termos econômicos, em classes que vão de A1 a E, conforme o total de pontos alcançados (de 0 a 34).

Uma versão do Parenting Stress Index (Abidin, 1995), o Índice de Estresse Parental - ISP, validada para o Brasil semanticamente e também em termos de conteúdo por Dessen e Bigras (2000), a partir da versão adaptada para Québec Canadá (Bigras \& LaFrenière, 1995). Esse tem a função de avaliar o componente de estresse presente nas interações pais-criança, considerando as características de temperamento e de comportamento da criança, a percepção dos pais a esse respeito, bem como as características de personalidade dos pais. É composto por 13 variáveis, sendo que quatro referem-se às características de temperamento da criança e compõem a dimensão Criança Difícil (adaptabilidade; exigência; humor; hiperatividade) e duas referem-se às expectativas dos pais em relação à criança, seja quanto à possibilidade de serem recompensados por ela, seja quanto à conformidade da criança a uma imagem idealizada (reforço; aceitabilidade), compondo a dimensão Interações Disfuncionais; três referem-se à personalidade dos pais (depressão, sentimento de competência e apego parental) e integram a dimensão Sofrimento Parental. Outras quatro variáveis são situacionais (a relação com o cônjuge; o apoio social disponível; a saúde e a restrição causada pela função parental), sendo essas que também integram a dimensão Sofrimento Parental.

O Inventário é constituído por 36 afirmações e as respostas ajustadas a uma escala do tipo Likert: concordo completamente (1), concordo (2), não tenho certeza (3), discordo (4), e discordo completamente (5). As respostas são corrigidas pontuando-as de forma invertida, ou seja, quem responde 1 pontua 5, 2 pontua 4 e assim por diante, sendo que a pontuação final pode variar de um mínimo de 94 pontos (baixo estresse) a um máximo de 117 pontos (alto estresse). Vale frisar que esse instrumento tem sido usado em outras investigações, no contexto brasileiro, mostrando-se bastante adequado (Dessen \& Szelbracikowski, 2004, 2006).

Questionário de Apoio Social - QAS, foi adaptado por pesquisadores do "Estudo Pró-Saúde" (Chor, Griep, Lopes \& Faerstein, 2001), a partir de uma outra medida, o Medical Outcomes Study - MOS, de Sherbourne e Stewart (1991). Este avalia o grau com que várias facetas do apoio são percebidas como de ajuda pelo indivíduo, sendo estas: a emocional, a de informação, a afetiva, a de interação positiva e a material. O instrumento também avalia a rede social, ou seja, a quantidade de pessoas com quem o indivíduo mantém contato. A versão adaptada por Chor e cols. (2001) é composta por vinte e quatro perguntas, sendo cinco relacionadas ao conceito de rede social e 19 ao de apoio social. As respostas dos participantes quanto ao apoio social são ajustadas a uma escala de freqüência: nunca (1), raramente (2), às vezes (3), quase sempre (4), sempre (5), sendo que a soma das respostas indica o nível de apoio total do participante, que pode variar de 19 a 95 pontos. Quanto às dimensões que compõem o Apoio Social, as referentes ao apoio Material, Emocional, de Informação e de Interação Social Positiva agregam quatro questões em cada uma delas, sendo que a pontuação mínima é 4 e a máxima é 20. Somente na dimensão de Apoio Afetivo, que agrega três questões, a pontuação mínima é 3 e a máxima é 15 . A dimensão de Apoio Material se refere à provisão de recursos e ajuda material; o Afetivo está ligado às demonstrações físicas de amor e afeto por parte de outras pessoas; o Emocional diz respeito às expressões de afeto positivo, de compreensão e sentimentos de confiança; o Apoio de Informação refere-se à disponibilidade de outras pessoas para fornecer orientações; e o de Interação Social Positiva se relaciona ao apoio recebido no sentido da interação e da disponibilidade das pessoas para a diversão e situações prazerosas.

\section{Procedimentos de coleta e de análise de dados}

O contato com os potenciais participantes, de ambos os grupos, foi realizado em suas residências, apresentando-lhes o Termo de Consentimento Livre e Esclarecido e solicitando-lhes a colaboração com o estudo. Na medida em que se obtinha o aceite, o participante era convidado a responder aos instrumentos imediatamente ou, dependendo de sua disponibilidade, agendava-se um outro dia e horário. No caso do G1 apresentou-se aos participantes uma carta que continha a autorização dos Conselhos Tutelares para contatá-los, ressaltando, entretanto, que o estudo não se atrelava às atribuições desse órgão, e que esse não teria acesso a nenhum tipo de informação vinculada aos casos individualmente.

As respostas fornecidas pelos participantes ao ISP e ao QAS foram corrigidas seguindo suas normas técnicas. As respostas fornecidas ao Questionário de Caracterização foram analisadas e convertidas em categorias numéricas, bem como a avaliação feita com a aplicação do CCEB. Todos os dados (sujeito a sujeito) foram compilados em uma planilha organizada no programa Windows Excell, de modo que pudessem ser analisados estatisticamente, visando averiguar diferenças significativas entre os grupos $(\mathrm{p}<0,05)$. Para tanto, utilizou-se o programa Jandel SigmaStat (Statistical Software 2.0), empregando-se o teste $t$ de Student para amostras independentes (quando a distribuição da variável era considerada normal) ou Mann-Whitney Rank Sum test (quando a distribuição não era normal).

\section{Resultados}

Pelos resultados da Tabela 1 nota-se que houve diferença significativa entre os grupos na dimensão "Criança Difícil" e no escore geral, indicando que os participantes do G1 vivenciam mais estresse relacionado à função parental do que os de G2, principalmente, devido à percepção que os mesmos possuem da criança (relativa às características de temperamento e de comportamento).

No Questionário de Apoio Social, especificamente no que se refere às dimensões Afetiva e de Interação Social Positiva, foram encontradas diferenças significativas entre os grupos, as quais indicaram um nível menor de apoio percebido para o G1. Além disso, o escore final também apresentou diferença nesta mesma direção, sendo possível afirmar que os participantes do G1 percebem-se com menos apoio social do que o G2. Com respeito ao número de parentes e amigos com os 
Tabela 1. Comparação dos grupos em relação às dimensões do ISP

\begin{tabular}{|c|c|c|c|c|c|}
\hline \multirow{2}{*}{ Dimensões } & \multicolumn{2}{|c|}{ G1 } & \multicolumn{2}{|c|}{ G2 } & \multirow[b]{2}{*}{$\mathrm{t}$} \\
\hline & Média & DP & Média & DP & \\
\hline Sofrimento Parental & 35,07 & 8,13 & 33,03 & 6,84 & ns \\
\hline Interações Disfuncionais & 31,33 & 8,25 & 28 & 5,61 & ns \\
\hline Criança Difícil & 34,13 & 7,58 & 29,7 & 6,65 & $2,40^{*}$ \\
\hline Escore final & 100,53 & 19,94 & 90,73 & 15,86 & $2,10^{*}$ \\
\hline
\end{tabular}

*p $\leq 0,05$

Tabela 2. Comparação dos grupos quanto às questões sobre rede social e referentes ao nível de apoio social

\begin{tabular}{|c|c|c|c|c|c|}
\hline \multirow{2}{*}{ Variáveis } & \multicolumn{2}{|c|}{ G1 $(n=30)$} & \multicolumn{2}{|c|}{$\mathrm{G} 2(\mathrm{n}=30$} & \multirow[b]{2}{*}{$\mathrm{t}$} \\
\hline & Média & DP & Média & DP & \\
\hline Rede de parentes & 1,9 & 1,65 & 2,27 & 1,74 & ns \\
\hline Rede de amigos & 1,57 & 2,01 & 1,3 & 1,12 & $\mathrm{~ns}$ \\
\hline Material & 14,10 & 4,96 & 15,90 & 4,25 & $\mathrm{~ns}$ \\
\hline Afetivo & 11,77 & 3,04 & 14,03 & 1,67 & $731,50 * *$ \\
\hline Emocional & 14,23 & 4,11 & 15,60 & 3,74 & ns \\
\hline Informação & 15,43 & 3,61 & 15,47 & 3,79 & $\mathrm{~ns}$ \\
\hline Interação Social Positiva & 13,67 & 4,23 & 17,00 & 3,34 & $-3,38 * * *$ \\
\hline Escore final & 69,20 & 17,3 & 78,00 & 12,55 & $780,50 *$ \\
\hline
\end{tabular}

${ }^{*} \mathrm{p} \leq 0,05 ; * * \mathrm{p} \leq 0,01 ; * * * \mathrm{p} \leq 0,001$

Tabela 3. Distribuição do número de participantes de ambos os grupos quanto à participação em atividades sociais

\begin{tabular}{lcccc}
\hline Variáveis & G1 & G2 & $\%$ \\
\hline Não participa de atividades de esporte ou cultura & $\mathrm{f}$ & $\%$ & $\mathrm{f}$ & 70 \\
Participa uma vez por semana & 24 & 80 & 21 & 20 \\
Participa de duas a três vezes por semana & 0 & 0 & 6 & 10 \\
Total & 6 & 20 & 3 & 100 \\
Não participa de reuniões de associações de moradores, sindica- & 30 & 100 & 30 & 80 \\
tos ou partidos & 25 & 83 & 24 & 7 \\
Participa uma vez por semana & 0 & 0 & 2 & 10 \\
Participa algumas vezes no ano & 4 & 13 & 3 & 3 \\
Participa uma vez no ano & 1 & 3 & 1 & 100 \\
Total & 30 & 100 & 30 & 74 \\
Não participa de trabalho voluntário & 24 & 80 & 22 & 3 \\
Participa uma vez por semana & 0 & 0 & 1 & 3 \\
Participa de duas a três vezes por semana & 0 & 0 & 1 & 3 \\
Participa algumas vezes no ano & 4 & 13 & 3 & 10 \\
Participa uma vez no ano & 2 & 7 & 3 & 10 \\
Total & 30 & 100 & 30 & 100 \\
\hline
\end{tabular}

quais há interação, destaca-se que não se observou diferença significativa entre os grupos.

Pelos resultados da Tabela 3 , observa-se que tanto no G1 como no G2, a maioria dos participantes não freqüenta ambientes sociais-comunitários de qualquer natureza. Nota-se, porém, em relação à participação em atividades esportivas e/ou culturais e em trabalhos voluntários, uma participação ligeiramente maior em G2.
Em paralelo a esses resultados, destaca-se que G1 se diferenciou de G2 na "impressão do bairro onde mora" $(t=755,00, p \leq 0,05)$, sendo que o primeiro grupo apresentou média inferior $(\mathrm{G} 1=6,93 \pm 2,56 ; \mathrm{G} 2=8,40 \pm 1,85)$, o que indica uma impressão menos favorável e um menor grau de satisfação com relação aos aspectos que envolvem o entorno comunitário, como por exemplo, a rede de serviços existentes naquele local. 


\section{Discussão}

A hipótese que norteou a presente investigação foi a de que o estresse parental e o apoio social seriam variáveis associadas ao abuso físico infantil, atuando, assim, em nosso contexto, como fator de risco. De modo geral, com base nos resultados encontrados, sobretudo os relativos aos escores finais obtidos com o ISP e o QAS, pode-se afirmar que a hipótese foi corroborada.

Numa análise mais detalhada, entretanto, percebe-se algumas nuances que oferecem pistas para refletir sobre os elementos do risco representado pelas variáveis em foco e de que modo esses se articulam na produção da problemática. Tratando, inicialmente, os resultados obtidos com o ISP, chama a atenção o fato de G1 ter se diferenciado de G2 especificamente na dimensão "Criança Difícil", sendo essa a que de fato contribui para o escore total, resultado compatível com os encontrados por DiLauro (2004). Isso significaria dizer que os pais notificados por abuso físico perceberiam as características referentes ao temperamento da criança e ao seu comportamento como uma fonte mais significativa de estresse do que os pais do grupo de comparação. De acordo com os indicadores inerentes ao ISP, os abusadores físicos tenderiam a perceber a criança como apresentando dificuldades para se adaptar a situações novas, demandando atenção e cuidado em demasia e de maneira inapropriada, e apresentando comportamentos que requerem um alto grau de vigilância (como distração, turbulência e dificuldade para realizar deveres escolares). Além disso, esses cuidadores tenderiam a sentir raiva ou ansiedade frente às variações de humor da criança, como choros excessivos e manifestações de tristeza (Bigras \& LaFrenière, 1995; Milner, 1994).

Assim, pode-se dizer que o estresse parental vivido por cuidadores que estão envolvidos em situações de abuso físico tem uma relação muito estreita com os processos cognitivos que subjazem à avaliação que fazem das características da criança, sejam essas ligadas a aspectos do temperamento ou do comportamento. Ainda que se padeça da falta de uma explicação robusta relativa ao mecanismo de interação entre o estresse e os padrões cognitivos apresentados por estes cuidadores, sabe-se que, de alguma forma, eles interagem para a composição do risco de abuso físico (Milner, 2003) e que o estresse parental é, provavelmente, a variável mais relevante nesse processo (Crouch \& Behl, 2001; Rodriguez \& Richardson, 2007; Rodriguez, 2010).

Não se pode, todavia, desprezar o fato de que a percepção negativa das características da criança contribui para a implementação de práticas parentais inadequadas, fundamentalmente coercitivas, e dessas terem produzido dificuldades comportamentais nas crianças, incrementando a experiência de estresse na relação cuidador-criança (Dopke \& Milner, 2000; Suzuki, 2010). Conforme a literatura, cuidadores de crianças com problemas de comportamento apresentam altos níveis de estresse (Dessen \& Szelbracikowski, 2004, 2006).

Ademais, é preciso considerar que as interações entre estresse, percepções negativas da criança e manejo inadequado de seu comportamento, em G1, remetem provavelmente a um processo em curso, de longa duração, pois se considera que nesse grupo o nascimento do primeiro filho se deu antes dos 20 anos, fato apontado pela literatura como indicador de risco para o abuso físico, já que esta variável, geralmente associada a outras, como nível sócio-econômico e educacional baixos, monoparentalidade combinada à falta de apoio social, bem como expectativas irreais com relação aos filhos, pode promover emoções negativas em relação ao papel parental (Combs-Orme \& cols., 2004). Com base nisso, há maior probabilidade de abuso infantil, sobretudo porque as interações com a criança, desde que essa é um bebê, tenderão a ser caracterizadas por uma menor responsividade por parte do cuidador, com menor engajamento verbal e físico, e pela apresentação de comportamento hostil e restritivo (Florsheim \& cols., 2003).

A não diferenciação dos grupos nas outras dimensões que compõem o ISP, Sofrimento Parental e Interações Disfuncionais, também remete a raciocínios sobre a especificidade dos elementos que parecem subjazer o abuso físico. Assim, numa primeira instância, deve-se lembrar que a dimensão Sofrimento Parental avalia especificamente características ligadas à personalidade dos pais, como a depressão, sentimentos de incompetência associados ao exercício do papel parental e de apego à criança, além de focar algumas variáveis situacionais (como o grau de restrição causada pela função parental e a qualidade da relação com o cônjuge). Na medida em que não se nota diferença significativa entre os grupos, pode-se pensar que os adultos que abusam fisicamente não se percebem como "tendo/sendo" eles mesmos uma fonte do problema. Alguns autores defendem que essa característica seria mais tipicamente encontrada em cuidadores avaliados como negligentes (Bazon, Mello, Bérgamo \& Faleiros, 2010; DiLauro, 2004).

Numa segunda instância, a dimensão Interações Disfuncionais, segundo a literatura, também se mostraria mais diretamente ligada ao fenômeno da negligência infantil (Bigras \& LaFrenière, 1995; Hildyard \& Wolfe, 2002). Lembrando que tal dimensão refere-se ao estresse proveniente de frustração quanto às expectativas com relação à criança, seja com respeito à possibilidade de ser recompensado por ela, seja com relação à sua conformidade a uma imagem idealizada, pode-se pensar que os abusadores, por não se diferenciarem nesse plano, teriam também a convicção de que podem conseguir da criança aquilo que "esperam dela". Dessa forma, pode-se supor que no repertório comportamental desses pais certamente figura a punição física como uma "ação educativa" para conseguir que a criança atenda às expectativas que têm, sendo que essa corre o risco de ser intensificada a cada inadequação infantil, aumentando também as chances de os abusos acontecerem.

Em síntese, considerando as três dimensões do ISP, pode-se pensar que a fonte de estresse, na percepção dos abusadores físicos, "é a criança", o problema está nela, sendo que eles mesmos se sentiriam capazes para conseguir "modificá-la" na direção de suas expectativas, não considerando ter eles mesmos qualquer dificuldade capaz de obstaculizar isso, nem ser a interação com a criança problemática em si, por mais coercitiva que esta se apresente. O quadro assim descrito encontra apoio em alguns estudos de abordagem qualitativa, nos quais se investiga os sentidos atribuídos por pais abusadores aos eventos de vitimização perpetrados aos filhos (Bazon, Matias \& Estevão, 2004; Oliveira, 2006).

Quanto ao apoio social, pode-se afirmar que no geral, os participantes de G1 disporiam de menos apoio que os de 
G2, o que indica ser esta variável um fator de risco para o abuso físico. No entanto, novamente, o detalhamento dos resultados oferece alguns elementos que permitem pensar quais aspectos do apoio social e de que modo esses operariam para a produção dos abusos físicos. Focalizando inicialmente os resultados que diferenciaram os grupos, duas dimensões relativas ao apoio social destacaram-se: a Afetiva, indicando que os participantes de G1 perceberiam com menos freqüência demonstrações físicas de amor/afeto por parte de outras pessoas; e a de Interação Social Positiva, indicando que eles se perceberiam com mais restrições quanto à vivência de situações sociais prazerosas/diversão. Os grupos não teriam se diferenciado no que concerne ao apoio de natureza material, informacional e emocional.

Um importante estudo de revisão sobre os fatores de risco para o abuso físico (Heyman \& Slep, 2000) indica a convergência da literatura no sentido de as famílias acometidas por essa problemática não se destacarem em termos de apoio instrumental, que abrangeria o apoio de natureza material e informacional. Ou seja, essas famílias não padeceriam da falta de ajuda quanto à provisão de recursos materiais (que pode incluir o financeiro), nem da oferta de orientações (que pode incluir os conselhos). Os problemas efetivamente residiram num plano mais subjetivo, referente aos aspectos relacionais atinentes aos sentimentos e às sensações.

Há, entretanto, uma nuance a ser feita com base nos resultados encontrados no que se refere ao apoio emocional (ligado a expressões verbais de afeto), onde os grupos não se diferenciaram, e ao apoio afetivo (ligado a demonstrações físicas de amor e afeto), onde houve diferença entre os grupos. Tais informações sugerem que as necessidades subjetivas dos cuidadores que cometem abusos físicos parecem requerer respostas que se dêem também no plano físico, pois nisso, parece prevalecer a insatisfação. Embora tal apontamento requeira investigações específicas e aprofundadas, este fornece pistas sobre a percepção que pais que maltratam fisicamente os filhos têm das relações sociais, parecendo essas serem para eles uma fonte de dificuldade, que gera sofrimento pessoal (Milner, 1994).

Já o resultado na dimensão de Interação Social Positiva pode estar indicando uma maior dificuldade dos participantes de G1 para disporem e experienciarem contextos de lazer e de relaxamento nos quais as relações sociais sejam satisfatórias ao ponto de gerarem sensações prazerosas. Nesta direção, ressalta-se que os participantes de G1 relataram mais insatisfação com o entorno comunitário do que os de G2, o que pôde ser verificado por meio da impressão relatada com relação ao bairro de moradia. Esses resultados podem ser refletidos à luz dos que foram encontrados por Gracia e Musito (2003), que verificaram uma integração social fragilizada para os cuidadores notificados por maus-tratos, sendo esta mediada por atitudes e sentimentos negativos quanto ao entorno social. Além disso, avaliar negativamente a comunidade de residência ou o entorno proximal, aumenta a sensação de ter pouco controle sobre os eventos sociais, o que incrementa o nível de estresse experimentado e, portanto, o risco de práticas parentais abusivas (Guterman \& cols., 2009).

É interessante notar que os grupos estudados não diferiram quanto ao número de pessoas que compõem suas redes sociais, bem como no nível de inserção em atividades sociais comunitárias, podendo-se afirmar que em ambos os grupos a participação em atividades e grupos é fraca. O que parece diferenciar os grupos, então, é a qualidade das experiências sociais. Estudos têm demonstrado que mais do que ter uma rede de pessoas disponíveis, que forneçam os recursos necessários, a percepção sobre a adequação do apoio recebido é o que se mostra mais relevante (Chor \& cols., 2001), sendo essa relação tanto mais evidente quanto mais a investigação é específica na área dos abusos infantis (Budd \& cols., 2000).

É preciso também considerar que a fraca integração social apresentada em ambos os grupos talvez indique a precariedade de oportunidades existentes na própria comunidade de residência dos participantes que, em sua maioria, pertencem a bairros ou comunidades populares, normalmente caracterizados por déficits em termos de quantidade e de qualidade de programas de lazer, cultura e serviços sociais. Desse modo, a avaliação dos grupos nessa dimensão talvez seja impertinente devido ao contexto, não sendo capaz de diferenciá-los.

Considerando os objetivos deste estudo, pode-se dizer que o mesmo corroborou apontamentos da literatura especializada e ainda ofereceu elementos novos para a análise de aspectos relacionados ao estresse e ao apoio social que parecem especificamente ligados ao fenômeno dos abusos físicos de crianças, denotando a complexidade da articulação que parece existir entre os fatores e o comportamento abusivo. Nesse sentido, contribuiu com pistas que poderão ser úteis na formatação de programas de prevenção ou de ajuda aos envolvidos nestas situações.

No entanto, novas pesquisas serão necessárias com vistas principalmente à investigação dos mecanismos pelos quais se estabelece a interação entre o estresse e o apoio social, com base em delineamento metodológico apropriado. Ademais, componentes diferenciados das variáveis focalizadas deverão ser integrados em pesquisas futuras, com o emprego de outros instrumentos. Por exemplo, níveis de estresse relacionados a eventos de vida problemáticos/adversos, como mudanças no status de emprego, problemas decorrentes de dificuldades financeiras, também parecem interferir nas práticas parentais implementadas (McCurdy, 2005) e precisam ser avaliados. Com relação ao apoio social, os recursos efetivamente disponibilizados aos indivíduos é uma dimensão importante de ser considerada em novas pesquisas, de modo a, inclusive, compreender melhor a percepção dos cuidadores quanto ao apoio que recebem. Considerando os parâmetros éticos, outras pesquisas também deverão abordar o problema dos abusos físicos longitudinalmente, devido à eminente necessidade de entender como esse problema aparece e se desenvolve em uma família.

\section{Referências}

ABEP - Associação Brasileira de Empresas de Pesquisa (2003). Critério de classificação econômica brasil. Retirado em 03/01/2006, de www.abep.org

Abidin, R. R. (1995). Parenting stress index. Odessa, FL: Psychological Assessment Resources.

Ateah, C. A. \& Durrant, J. E. (2005). Maternal use of physical punishment in response to child misbehavior: implications for child abuse prevention. Child Abuse \& Neglect, 29, 169-185. 
Balge, K. A. \& Milner, J. S. (2000). Emotion recognition ability in mothers at high and low risk for child physical abuse. Child Abuse \& Neglect, 10 (24), 1289-1298.

Bazon, M. R.; Matias, A.S.A., \& Estevão, R. (2004). Domestic violence against children and adolescents: the actors perspective. In: Victimization of children and youth: an international research conference. Portsmouth Program, 14-14.

Bazon, M. R., Mello, I. L. A., Bérgamo, L. P. D., \& Faleiros, J. M. (2010). Negligência infantil: Estudo comparativo do nível socioeconômico, estresse parental e apoio social. Temas em Psicologia, 18(1), 71-84.

Bigras, M., \& Lafrenière, P. J. (1995). Índice de stress parental. Manuel d'utilisation. Complément francophone à l'édition américaine. Multi-Healthy System.

Bigras, M., \& Paquette, D. (2000). L'Interdépendance entre les sous-systèmes conjugal et parental: Une analyse personneprocessus-contexte. Psicologia: Teoria e Pesquisa, 16(2), 91-102.

Bordin, I. A. S., Paula, C. S., Nascimento, R., \& Duarte, C. S. (2006). Severe physical punishment and mental health problems in an economically disadvantaged population of children and adolescents. Revista Brasileira de Psiquiatria, 28(4), 290-296.

Bringiotti, M. I. (1999). Maltrato infantil. Factores de riesgo para el maltrato físico en la poblacion infantil que concurre a las escuelas dependientes del Gobierno de la Ciudad de Buenos Aires. Madrid/Buenos Aires: Niño y Dávila editores.

Budd, K. S., Heilman, N. E., \& Kane, D. (2000). Psychosocial correlates of child abuse potential in multiply disadvantaged adolescent mothers. Child Abuse \& Neglect, 5(24), 611-625.

Carmo, C. J., \& Harada, M. J. C. S. (2006). Violência física como prática educativa. Revista Latino-Americana de Enfermagem, 14(6), 849-856.

Cecconello, A. M., De Antoni, C., \& Koller, S. H. (2003). Práticas educativas, estilos parentais e abuso físico no contexto familiar. Psicologia em Estudo, 8, 45-54.

Chor, D., Griep, R. H., Lopes, C. S., \& Faerstein, E. (2001). Medidas de rede e apoio social no Estudo Pró-Saúde: pré-testes e estudo piloto. Cadernos de Saúde Pública, 4(17), 887-896.

Combs-Orme, T., Cain, D. S., \& Wilson, E. E. (2004). Do maternal concerns at delivery predict parenting stress during infancy? Child Abuse \& Neglect, 28, 377-392.

Coohey, C. (1996). Child maltreatment: Testing the social isolation hypothesis. Child Abuse \& Neglect, 20(3), 241-254.

Crouch, J. L., \& Behl, L. E. (2001). Relationships among parental beliefs in corporal punishment, reported stress, and physical child abuse potential. Child Abuse \& Neglect, 25, 413-419.

Crouch J. L., Milner, J. S., \& Thomsen, C. (2001). Childhood physical abuse, early social support, and risk for maltreatment: current social support as a mediator of risk for child physical abuse. Child Abuse \& Neglect, 25, 93-107.

De Antoni, C., Barone, L. R., \& Koller, S. H. (2007). Indicadores de risco e proteção em famílias fisicamente abusivas. Psicologia: Teoria e Pesquisa, 23(2), 125-132.

Dessen, M. A., \& Bigras, M. (2000). As crianças pré-escolares com problemas de comportamento e suas famílias: identificação de fatores de risco. Relatório de Pesquisa. Brasília:CNPq.

Dessen, M. A., \& Braz, M. P. (2000). Rede social de apoio durante transições familiares decorrentes do nascimento de filhos. Psicologia: Teoria e Pesquisa, 16(3), 221-231.
Dessen, M. A., \& Szelbracikowski, A. C. (2004). Crianças com problemas de comportamento exteriorizado e a dinâmica familiar. Interação em Psicologia, 8(2), 171-180.

Dessen, M. A., \& Szelbracikowski, A. C. (2006). Estabilidades e mudanças em padrões familiares de crianças com problemas de comportamento exteriorizado. Paidéia, 16(33), 71-80.

De Paúl, J., \& Domenech, L. (2000). Childhood history of abuse and child abuse potential in adolescent mothers: a longitudinal study. Child Abuse \& Neglect, 5(24), 701-713.

DiLauro, M. D. (2004). Psychosocial factors associated with types of child maltreatment. Child Welfare, 83, 69-99.

Dopke, C. A., \& Milner, J. S. (2000). Impact of child noncompliance on stress appraisals, attributions, and disciplinary choices in mothers at high and low risk for child physical abuse. Child Abuse \& Neglect, 4(24), 493-504.

Florsheim, P., Sumida, E., McCann, C., Fukui, R., Seedfeldt, T., Winstanley, M., \& Moore, D. (2003). The transition to parenthood among young African American and latino couples: relational predictors of risk for parental dysfunction. Journal of Family Psychology, 17(1), 65-79.

Gracia, E., \& Musitu, G. (2003). Social isolation from communities and child maltreatment: a cross-cultural comparison. Child Abuse \& Neglect, 27, 153-168.

Guterman, N. B., Lee, S. J., Taylor, C.A., \& Rathouz, P. J. (2009). Parental perceptions of neighborhood processes, stress, personal control, and risk for physical child abuse and neglect. Child Abuse \& Neglect, 33, 897-906.

Hecht, D. B., \& Hansen, D. J. (2001). The environment of child maltreatment: contextual factors and the development of psychopathology. Aggression and Violent Behavior, 6, 433457.

Heyman, R. E., \& Slep, A. M. S. (2000). Risk factors for family violence: introduction to the special series. Aggression and Violent Behavior, 6, 115-119.

Hildyard, K. L., \& Wolfe, D. A. (2002). Child neglect: developmental issues and outcomes. Child Abuse \& Neglect, 26, 679-695.

Jackson, A.P. (2000). Maternal self-efficacy and children's influence on stress and parenting among single black mothers in poverty. Journal of Family Issues, 21(1), 3-16.

Lee, Y. (2009). Early motherhood and harsh parenting: The role of human, social, and cultural capital. Child Abuse \& Neglect, $33,625-637$.

McCurdy, K. (2005). The influence of support and stress on maternal attitudes. Child Abuse \& Neglect, 29, 251-268.

McLewin, L. A., \& Muller, R. T. (2006). Attachment and social support in the prediction of psychopathology among young adults with and without a history of physical maltreatment. Child Abuse \& Neglect, 30, 171-191.

Milner, J. S. (1994). Assessing physical child abuse risk: the child abuse potential inventory. Clinical Psychology Review, 6(14), 547-583.

Milner, J. S. (2003). Social information processing in high-risk and physically abusive parents. Child Abuse \& Neglect, 27, 7-20.

Montes, M. P., De Paúl, J., \& Milner, J. S. (2001). Evaluations, attributions, affect, and disciplinary choices in mothers at high and low risk for child physical abuse. Child Abuse \& Neglect, 25, 1015-1036.

Ochotorena, J. P., \& Madariaga, M. I. A. (1996). Manual de Protección Infantil. Barcelona: Masson. 
Oliveira, T. T. S. S. (2006). Educar é punir? Compreendendo pontos de vistas de pais denunciados por violência física contra seus filhos. Dissertação de Mestrado, Universidade de São Paulo, Ribeirão Preto, SP.

Organização Mundial de Saúde \& ISPCAN (2006). Preventing child maltreatment: A guide to taking action and generating evidence. New York: WHO Press.

Palusci, V. J., Smith, E. G., \& Paneth, N. (2005). Predicting and responding to physical abuse in young children using NCANDS. Children and Youth Services Review, 27, 667-682.

Reichenheim, M. E., Dias, A. S., \& Moraes, C. L. (2006). Coocorrência de violência física conjugal e contra filhos em serviços de saúde. Revista de Saúde Pública, 40(4), 595-603.

Rodriguez, C. M. (2010). Personal contextual characteristics and cognitions: Predicting child abuse and disciplinary style. Journal of Interpersonal Violence, 25(2), 315-355.

Rodrigues, C. M., \& Richardson, M. J. (2007). Stress and anger as contextual factors and preexisting cognitive schemas: Predicting parental child maltreatment risk. Child Maltreatment, 12(4), 325-337.

Sacroisky, G. (2003). Maltrato físico: um problema de salud que nos involucra. Archivos Argentinos de Pediatria, 101(1), 64-72.

Sherbourne, C. D., \& Stewart, A. L. (1991). The MOS social support survey. Social Science and Medicine, 38, 705-714.
Suzuki, S. (2010). The effects of marital support, social network support, and parenting stress on parenting: self-efficacy among mothers of young children in Japan. Journal of Early Childhood Research, 8(1), 40-66.

Weber, L. N. D., Viezzer, A. P., \& Brandenburg, O. J. (2004). O uso de palmadas e surras como prática educativa. Estudos de Psicologia, 9(2), 227-237.

Woodward, L. J., \& Fergusson, D. M. (2002). Parent, child, and contextual predictors of childhood physical punishment. Infant and Child Development, 11, 213-235.

Zelenko, M. A., Huffman, L., Lock, J., Kennedy, Q., \& Steiner, H. (2001). Poor adolescent expectant mothers: can we assess their potential for child abuse? Journal of Adolescent Health, $29,271-27$.

\section{Curso Intensivo de Terapia Sexual (CITS)}

Local: Salvador - BA

Período: 2 a 15 de janeiro de 2011 